\title{
Erste Schritte auf dem Weg zur modernen Psychologie?
}

\section{Oder wie Karl Philipp Moritz' Erfahrungsseelenkunde den Gegenstand einer neuen Wissenschaft bestimmt}

\author{
Monique Lathan
}

Journal für Psychologie, 26(1), 30-48

https://doi.org/10.30820/8247.03

www.journal-fuer-psychologie.de

\section{Zusammenfassung}

Seit den 1970er Jahren ist die Psychologie eine der am stärksten expandierenden wissenschaftlichen Disziplinen. Auch ist ihr Gegenstand, die Psyche, aus alltäglichen wie medienöffentlichen Diskursen nicht mehr wegzudenken. Doch wie kam dieser Gegenstand in die Welt? Ein genauerer Blick auf die Anfänge der sich Ende des 18. Jahrhunderts entwickelnden Psychologie zeigt, dass die wissenschaftliche Konstruktion der Seele einer Logik folgt, die bis heute erfolgreich zu sein scheint. Menschliches Handeln scheint darin nicht schlicht einem je konkreten Willensinhalt zu folgen, der sich Gründen und Zwecken verdankt, die sich das Individuum setzt. Vielmehr werden hinter allen willentlichen Akten zugleich ganz unwillentliche Prozesse vermutet und es wird versucht, ihnen als das Handeln leitende Kräfte auf die Spur zu kommen. So etabliert und selbstverständlich dieser Gedanke gerade heute scheinen mag, so weitreichend sind die ihm eigenen Implikationen und Konsequenzen für die theoretische Bestimmung der menschlichen Individualität. Diese Logik bloßzulegen nimmt sich der Artikel am Beispiel von Karl Philipp Moritz' Erfahrungsseelenkunde vor.

Schlüsselwörter: Wissenschaft, Psychologie, Psyche, Psychologiegeschichte, Erfahrungsseelenkunde, Karl Philipp Moritz, Aufklärung

\section{Summary}

First steps on the way to modern psychology? Or how Moritz's

Erfahrungsseelenkunde identifies the subject of a new science

Since the 1970s, psychology has been one of the most expanding scientific disciplines. Also its object, the psyche, has become an integral part of everyday and media discourses. But how has this object appeared in the world? A closer look at the beginnings of psychology at the end of the $18^{\text {th }}$ century reveals that the scientific construction of the psyche follows an up to 
day successful logic. Accordingly, human action appears not to simply flow from a concrete will with a rationale and a purpose set by the individual. Rather, behind deliberative acts nondeliberative processes are suspected, and are sought to be revealed as action-guiding forces. As established and self-evident as this thought nowadays appears, as far-reaching are its immanent implications and consequences for the theoretical definition of human individuality. In taking Karl Philipp Moritz’ »Erfahrungsseelenkunde « as an example, this article seeks to disclose this logic.

Keywords: science, psychology, psyche, history of psychology, construction of the psyche, experiential psychology, Karl Philipp Moritz, enlightenment

\section{Die Psyche wird entdeckt. Einleitung}

Die Psyche ist gegenwärtig wohl einer der beliebtesten Gegenstände wissenschaftlicher, medienöffentlicher wie alltäglicher Diskurse - keine Diskussion über Fremdenfeindlichkeit ohne die psychologische Erklärung der Angst vor dem Fremden, keine Partnervermittlung ohne Persönlichkeitstest, kein Berufserfolg ohne intrinsische Motivation etc. So vielfältig die Bezüge sind, in denen die Psyche als Grund und Begründung auftritt, so wenig wird an ihrer Existenz gezweifelt. ${ }^{1}$ Das war nicht immer so selbstverständlich.

Nach dem Innenleben des Menschen mit eigenen wirklichkeitsstiftenden Gesetzmäßigkeiten galt es zunächst überhaupt erst zu suchen, ihm musste eine Wirkmacht zugeordnet werden, die umrissen und spezifiziert werden wollte. Entlang des Anspruchs, die Psyche systematisch in ihren Gesetzmäßigkeiten zu erklären, entwickelte sich Ende des 18. Jahrhunderts die psychologische Wissenschaft. ${ }^{2}$ Sie trat an, die Psyche als eigenes Wesen rein empirisch zu bestimmen - »Fakta, und kein moralisches Geschwätz « (Moritz, 1783, S. 8) sollten inhaltlich dafür bürgen. Das Magazin zur Erfahrungsseelenkunde, das als erste und seinerzeit wichtigste psychologische Zeitschrift Deutschlands von 1783 bis 1793 erschien, leitete Karl Philipp Moritz entsprechend in der Metaphorik absoluten Nichtwissens um den Gegenstand ein: » welche unbetretne Pfade, welche Dunkelheit, welch ein Labyrinth! « (ebd., S. 7) Ob der zu erforschende Gegenstand, die menschliche Psyche, über den man doch eingestandenermaßen noch gar nichts wusste, überhaupt existiert, schien dennoch nicht in Zweifel zu stehen.

»Wie leicht kann hier ein falscher Tritt, den Suchenden irre führen, daß er sein ganzes Leben hindurch nach einem Blendwerke hascht, und nie den milden Strahl der Wahrheit findet, welcher nur den beglückt, der an der Hand der Vernunft geleitet, gleichfern von Enthusiasmus und Kälte, den Weg der ruhigen Weisheit wandelt « (ebd.). 
Diesen aufklärerischen Anspruch, der immerhin kein geringerer ist, als die Wahrheit über die Psyche als (eine) treibende Kraft der menschlichen Existenz zu finden, um erhellende Erkenntnis über diese zu generieren, konstruiert Moritz expressis verbis (selbst). Denn in der Darstellung des Gegenstandes als Rätsel, dessen Lösung einer Wissenschaft bedürfe, erscheint im Gestus von Wissenschaftlichkeit eine Objektivität des Gegenstandes, die lediglich sprachlich erzeugt ist. Das heißt nur dadurch, dass Moritz rhetorisch die Erhellung des Dunkels per vernünftiger Wahrheitssuche in Aussicht stellt, ergibt sich der zu erhellende Gegenstand. Durch das Bemühen von Dichotomien zur Beschreibung seines gedanklichen Konstrukts, der Psyche, setzt er dieses in einen maximalen Gegensatz zum rationalen zeitgenössischen Erkenntnisstand, verleiht ihm so die Eigenschaft des Rätselhaften und qualifiziert es mithin zum Objekt wissenschaftlichen Interesses. Mit diesen Oppositionen kreiert er den Gegenstand seiner Untersuchung, zugleich aber erwächst aus den dichotom gesetzten Abstraktionen hell vs. Dunkel und irre vs. vernünftig gerade kein konkretes Forschungsobjekt. Stattdessen soll die Sache, ihre Existenz, im Modus der Darstellung, im »Wie« der Schilderung und nicht im Ausrichten auf ein »Was «, das zu schildern wäre, glaubhaft werden.

Darin zeigt sich der stilistisch konstruktive Ertrag der Metaphorik Moritz' - Kraft der Tatsache, dass er nichts über die Sache der Seele weiß, Respekt vor diesem Dunkel hat, ergibt sich überhaupt erst die Sache. Oder noch einmal anders ausgedrückt: Die Seele als das zu erforschende Feld wird gewissermaßen nur behauptet, bleibt aber nichtsdestoweniger Bezugs- und Ausgangspunkt der Seelenkunde. Bei näherer Betrachtung wird sich zeigen, wie dieses Forschungsvorhaben bereits in seiner Anlage noch weitere theoretische Unterstellungen enthält, die Moritz ex post aus seinem empirischen Material wieder herausliest.

Der folgende Beitrag nimmt sich vor, diese Unterstellungen anhand von Auszügen aus Moritz' Magazin zur Erfahrungsseelenkunde zu rekonstruieren und deren immanente Logik und damit ihren inneren Zusammenhang zu entwickeln. Im Ergebnis zielt die Analyse somit auf eine Explikation des Moritzschen Seelenbegriffs wie auch seines Beweisverfahrens.

\section{Ein Interesse erschafft sich seinen Gegenstand: Moritz' Relevanzbehauptung}

Moritz kündigt sein Vorhaben, eine Erfahrungsseelenkunde zu entwerfen, 1782 in der Zeitschrift Deutsches Museum an. Mit dem Versuch, zunächst die Relevanz eines solchen Magazins zu begründen, konstatiert er, dass die menschliche Seele bis dato nicht genügend Beachtung fand. 
»Unter allen übrigen Dingen hat der Mensch sich selber seiner eignen Aufmerksamkeit vielleicht noch am allerwenigsten werth gehalten. Blos weil das dringendste Bedürfniß der Krankheit ihn dazu nöthigte, fing er an, seinen Körper genauer kennen zu lernen. Weil er dieses Bedürfnis bei den Krankheiten der Seele nicht so lebhaft empfand, so vernachlässigte er auch die Kentniß dieses edelsten Teils seiner selbst« (Moritz, 1782, S. 485f.).

Moritz gibt damit zunächst einmal überhaupt zu Protokoll, was er sich als seinen Forschungsgegenstand auswählt und wie er diesen auffasst: »Der Mensch« als AbstraktAllgemeines, als Gattungswesen, zerfalle, so viel hält Moritz trotz aller Unkenntnis doch für gesichert, in zwei Teile, nämlich den Körper und die Seele. Letztere ist es, mit deren Erforschung er sich befassen will. Damit ist der erste und ein wesentlicher Schritt zur Begründung der neuen Wissenschaft geleistet: ihr Gegenstand ist in der Welt beziehungsweise ist in die Welt hineinbehauptet, denn dass es das Ding namens Seele gibt, von dem man eingestandenermaßen keine Kenntnis hat, firmiert zumindest an dieser Stelle als Ausgangspunkt und Setzung, hinter die die Reflexion nicht zurücktritt. Moritz begründet im nächsten Schritt, wieso bisher alles Wissen über diesen Gegenstand fehlt und gibt hierdurch implizit bereits nähere Bestimmungen von ihm: Wie der Körper, so befände sich auch die Seele normalerweise in einem Zustand der Gesundheit, der jedoch von Krankheiten gestört werden könne. In beiden Fällen sei es dann die richtige Kenntnis des betroffenen Teils, die seine Heilung ermögliche. Diese Bestimmungen sowie ihre Plausibilität und Anschauung werden jedoch gar nicht aus dem Gegenstand begründet, sondern durch den Vergleich erzeugt: So wie jeder seinen Körper kennt, so soll er sich auch (s)eine Seele vorstellen, als Sache, gegenständlich in der Welt vorfindbar, mit bestimmten Beschaffenheiten und Gesetzmäßigkeiten, die jedoch nicht gleich der Kenntnis zugänglich, sondern erst durch wissenschaftliche Erforschung zu gewinnen sind. Diese Erforschung sei bisher versäumt worden und soll nun nachgeholt werden. Für die Begründung seines Desiderats hilft Moritz wieder der Vergleich: Aus der diagnostizierten Irrelevanz der Seele im Hinblick auf ein bis dahin (unverständlicherweise) fehlendes Bedürfnis, diese zu erkunden - einer Rücksichtnahme auf sie wurde kaum wertgehalten - just ihre Relevanz ableiten zu wollen, entbehrt sachlich jeder Grundlage. Vielmehr formuliert Moritz umgekehrt seinen Willen zu solch einer wissenschaftlichen Erfkundung der Seele und stiftet diesem bloß sprachlich inszenierten Erfordernis über besagten Vergleich zwischen Körperkrankheit und Seelenkrankheit einen Inhalt. Das heißt daraus, dass aus dem Mangel (Krankheit) eines anderen Gegenstandes (des Körpers) ein Untersuchungsbedürfnis entstanden war, begründe sich die Notwendigkeit der Betrachtung dieses Gegenstandes (der Seele), der - obwohl Moritz' Behauptung nach edelster Teil des Menschen - diese aus sich selbst heraus nicht erzeuge. Diese Begründungslogik wird nicht ob ihrer Widersprüchlich- 
keit aufgegeben, sondern wird ganz im Gegenteil aufgrund seiner inszenierten Analogie zwischen Körper- und Seelenkrankheit zum Sorgentitel nach bewährtem Muster, der sich nunmehr auf die Psyche als abstrakt gefasster Inhalt der Seele bezieht. Auf diese Weise wird die Psyche bei Moritz zum Ausgangspunkt der Relevanzbegründung für eine Erfahrungsseelenkunde, eine systematische Erforschung der Seelenempirie herangezogen. Die leere Seite des angestellten Vergleichs, der die Seele betrifft, insofern sie erst empirischer Gegenstand werden soll, wird gefüllt mit der Beobachtung, dass viele Individuen als Teile der gesamten Gesellschaft gegen dieselbe - obgleich sie deren Glieder sind - verstoßen.

»Tausend Verbrecher sahen wir hinrichten, ohne den moralischen Schaden dieser, von dem Körper der menschlichen Gesellschaft abgesonderten Glieder unserer Untersuchung wert zu halten. Da diese doch ein ebenso wichtiger Gegenstand für den moralischen Arzt und für den nachdenkenden Philosophen, als für den Richter ist, welcher die traurige Operation veranstalten muß (ebd., S. 486).

Am Verbrechen und seiner härtesten rechtlichen Konsequenz, der Hinrichtung des Verbrechers, will Moritz die weitreichende Relevanz seines Gegenstandes aufzeigen und beglaubigen - und liefert damit weitere Bestimmungen über die Seele und deren Zusammenhang mit ihresgleichen, der Gesellschaft: Mit dem Verweis auf den Schaden aller durch den Verstoß einzelner wird einerseits die Seele als edelster Teil des Menschen und basale Voraussetzung seiner selbst plausibilisiert und andererseits der Maßstab für die Existenz der Seele gesetzt, der nicht von einem Urteil über sie zu trennen ist. Dass es die Seele in ihrer Maßgeblichkeit für menschliche Handlungshorizonte gibt, macht sich besonders deutlich geltend an den durch sie verursachten Verstößen gegen die Gesellschaft (Verbrechen): Dass ihre Existenz am Verstoß gemessen ist, weist ihr implizit ein Sollen, einen eigentlichen mehr oder weniger realisierten Zustand zu. Mit der Bestimmung der Einzelseele als immer zugleich Bestandteil des Ganzen (der Gesellschaft, des Menschen im Allgemeinen) als Abstraktion vom Konkret-Einzelnen ist mitgesagt, dass sowohl das Gelingen als auch die Verhinderung eines moralischen Miteinanders seinen Grund im Individuum (der Gesundheit/Krankheit seiner Seele) hat. Unter der Hand sind damit zwei Identifikationen geleistet: Zum einen ist das geltende Recht gleichgesetzt mit der Moral und als Maßstab für diese gegeben und der Verstoß gegen das Recht als moralischer Schaden gekennzeichnet. Zum anderen ist die Moral mit der Seele identifiziert beziehungsweise als Indikator für deren Zustand behauptet. Konsequenterweise nötigt dieses Ideal einer eigentlich moralisch verfassten Gesellschaft, Moritz' Argumentation folgend, die systematische Untersuchung der Einzelseele auf.

In der Identifikation mit ihrem Sollenszustand entsteht die Notwendigkeit eines 
empirischen Zugangs als der anderen Seite einer bloß abstrakt-allgemein behaupteten Seele, gegen die Moritz polemisiert, wenn er sagt:

»Was mich darüber beruhiget, daß ich die gegenwärtige Sündfluth von Büchern noch mit einem neuen Buche vermehren will, ist dieses, daß ich Fakta, und kein moralisches Geschwätz, keinen Roman, und keine Komödie, liefere, auch keine andern Bücher ausschreibe (Moritz, 1783, S. 8).

So sichert Moritz die Notwendigkeit (s)eines Untersuchungsvorhabens zum Seelenzustand beziehungsweise der Psyche des Individuums, das er einerseits als aufklärerisches Erfordernis des Menschen und andererseits als eigene innovative Herausforderung bestimmt, zusätzlich über die Diagnose des Mangels an längst überfälligen, angemessen wissenschaftlichen Zugängen ab.

\section{Die Seele als Schlüssel zur idealen Gesellschaft: Moritz' Forschungsnotwendigkeit}

Sein behauptetes Anliegen, ganz ohne theoretisches Vorurteil, rein empirisch Fakten über die Seele zu sammeln, steht jedoch nicht nur im Widerspruch zu den eben genannten Bestimmungen, die Moritz noch vor jeder Untersuchung für selbstverständlich befindet, sondern auch dazu, dass im Vorfeld schon feststeht, wessen Betätigungsfeld die Seele mit ihren Schäden ist: »Da diese doch ein ebenso wichtiger Gegenstand für den moralischen Arzt und für den nachdenkenden Philosophen, als für den Richter ist, welcher die traurige Operation veranstalten muß (ebd., 1782, S. 486). Neben dem Richter als exekutive Repräsentanz geltenden Rechts sei die kranke Seele, die zum Beispiel verbrecherisches Handeln verursachen kann, Gegenstand des moralischen Arztes wie des Philosophen. Dass der Arzt als einer der Moral und ein Philosoph, der für die Konstruktion allgemeiner Weltzusammenhänge steht, ebenfalls Adressaten von seelischen Beeinträchtigungen sein sollen, verweist darauf, dass die Seele im Vergleich zum Körper von vornherein nicht nur im Verhältnis zu sich gedacht wird, sondern in einem ihr äußerlichen steht - allgemein zur Welt, konkret zur vorherrschenden Gesellschaft. Wenn es das Charakteristikum der Seele ist, dass sie ein Verhältnis von Individuum und seinen gesellschaftlichen Bedingungen ist, dann ist der Möglichkeit nach eine Entsprechung von Individuum und Gesellschaft unterstellt. ${ }^{3}$

»Vom Lebhaftesten unter diesen bis zum Trägsten; von der feinsten Organisation bis zur gröbsten; vom feurigsten Blick bis zum kältesten; und von der aufstrebendsten Stärke bis 
zur hinfälligsten Schwäche - und doch dies alles nur verhältnismässige Begriffe - jeder ist gut, und kann gut sein, in seiner Art « (ebd., S. 501).

So ist jedes Subjekt »in seiner Art «, also in potentia, gut beziehungsweise moralisch, auch wenn es im konkreten Fall davon abweicht.

Die bisherigen Überlegungen zusammengenommen lassen den Schluss zu, dass nach Moritz eine Gesellschaft - als über Moral konstituierte Einheit von Einzelindividuen - aus dem Individuum selbst hervorgeht, mithin in ihm bereits angelegt ist. Entsprechend gehen dann auch die moralischen Schäden am Gesamtkörper aus dem Subjekt hervor. Somit ist das Subjekt der Grund für die noch nicht realisierte, über Moral zusammengehaltene Gesellschaft und steht zugleich, insofern es in potentia gut ist, für die perspektivische Realisation derselben. So sieht Moritz offenbar den Zweck des geplanten Magazins in der in Aussicht gestellten Verwirklichung der Einheit zwischen Individuum und gesellschaftlichen, pauschal positiv gedachten Zwecken.

Den in der Vorstellung eines aus der menschlichen Natur resultierenden Rechts enthaltenen Widerspruch allerdings, nämlich dass etwas, das aus der Natur des Menschen kommt, anschließend gewaltsam gegen ihn durchgesetzt werden muss (kodifiziertes Recht und Strafe), löst Moritz hier zu Lasten des Einzelindividuums auf: Die Abweichung vom Recht ist in der gestörten Seele begründet, ist mithin ein Verstoß gegen ihre eigentliche Moralität - und damit gegen sich selbst. Doch mit der Auflösung des Rätsels »Abstraktum Mensch « vermittels seiner Seelenkunde über eine durch Moral konstituierte Gesellschaft begibt sich Moritz in einen zweiten Widerspruch. Denn Moral, als Prinzip zur Zurücknahme eigener Interessen zugunsten anderer beziehungsweise der Gemeinschaft, kann logisch nicht die positive Bestimmung der zu erklärenden Sache (Gesellschaft) abgeben - so als würde sich gerade über Nicht-Handeln ein Handlungszusammenhang herstellen. So erklärt Moritz beide, die Individualseele und die Gesellschaft, ganz zirkulär aus dem je anderen und erhält damit zwei leere Begriffe: Die Seelentätigkeit wird gefasst als Entsprechung oder Abweichung von gesellschaftlichen Vorgaben, die ihrerseits jedoch zugleich Ausdruck individueller Seelentätigkeit sein sollen. Aus der Logik dieses behaupteten Zusammenhangs leitet Moritz die Notwendigkeit der Erforschung der Seele ab:

»Alle diese Beobachtungen erstlich unter gewissen Rubriken in einem dazu bestimmten Magazine gesamelt, nicht eher Reflexionen angestellt, bis eine hinlängliche Anzahl Fakta da sind, und dann am Ende dies alles einmal zu einem zweckmäßigen Ganzen geordnet, welch ein wichtiges Werk für die Menschheit könte dies werden! Das wäre noch der einzige Weg, wie das menschliche Geschlecht durch sich selber mit sich selber bekannter werden, und sich zu einem höhern Grade der Vollkommenheit empor schwingen könte, so wie ein einzelner Mensch durch Erkenntniß seiner selbst vollkommener wird « (ebd.). 
Im Umkehrschluss ist die Menschheit an ihrer Vollkommenheit gehindert solange es an Wissen über die Individualseele mangelt, weil dort die Potenz zur vollkommenen Moralität verborgen liegt. Bemerkt sei an dieser Stelle noch, wie sich die eingangs getätigte Abstraktion »der Mensch « in Moritz' Bestimmung von Gesellschaft fortschreibt. Wird letztere nämlich gefasst als Zusammenschluss von »Menschen « so wird damit explizit abgesehen von allen Spezifika und sozialen Charakteren, die die verschiedenen Menschen in einer gegebenen Gesellschaft einnehmen, mithin von der gesellschaftlichen Funktion, die sie darin vollziehen und den Folgen, die das hat - es wird nur noch ihr abstrakt gemeinsames Menschsein ins Auge gefasst. Gesellschaft ist damit gefasst als gemeinsames Anliegen und Werk von Gleichen und ein Verstoß gegen selbige seitens eines Individuums - egal mit welchem Inhalt - ist nicht ein Verstoß gegen ein ihm äußerliches, womöglich ihm gegensätzliches Prinzip, sondern ein Verstoß des Individuums gegen sich selbst und sein Menschsein; dies ist die enorme legitimatorische Leistung einer ganz unscheinbaren Abstraktion.

Jaeger und Staeuble (1978) hingegen sehen im Zusammentragen der individuellen Lebensgeschichten und ihrer nachträglichen Systematisierung, insofern es dabei »nicht um äußere Verhaltensdaten geht [...], sondern um den inneren Zusammenhang der psychischen Geschichte « (S. 77), eine historisch ermöglichte »Denkstruktur « (ebd.), die ein In-Distanz-Treten des Individuums zu seinen gesellschaftlichen Bedingungen zeigt. So deuten sie Moritz' frühe empirische Psychologie als geistiges oder diskursives Abziehbild der politökonomischen Veränderungen jener Zeit. Unberücksichtigt bleibt dabei aber die oben dargestellte Eigenlogik des Moritzschen Vorhabens, in der gerade äußere Verhaltensdaten (etwa verbrecherisches Handeln) zum Ausgangspunkt der Theorie innerpsychischer Willensdeterminanten herangezogen werden. Im Sinne Jaegers und Staeubles ließe sich vor diesem Hintergrund weiterfragen, weshalb, wie Spies (1992) annimmt, der für die moderne Psychologie konstitutive Gedanke, »menschlich[es] Verhalten als Resultante aus dem Wirken seelischer Instanzen « (ebd., S. 125) zu begreifen, mit der Herausbildung der bürgerlichen Gesellschaft aufkommt.

\section{Die Erforschung der Seele zeigt, dass es sie gibt! Moritz' Konstruktion eines determinierten Bewusstseins beziehungsweise relativierten Willens}

Moritz macht sich im Folgenden daran, die Seele aufzufinden, und zwar dadurch, dass er Phänomene aufzeigt, die er als Wirkungen interpretiert, um von diesen dann auf die Seele als deren Verursacherin zurückzuschließen. An diesen Fällen wird deutlich, welchen Stellenwert Moritz dem bewussten Willen im Verhältnis zu den von ihm geschilderten Seelenphänomenen zumisst. Wie die Seele über ihre Wirkungen gefunden 
werden soll, sollen die folgenden Analysen einzelner Fallbeispiele aus dem Magazin zur Erfahrungsseelenkunde zeigen, die ihrer Argumentationslogik nach das Magazin durchziehen. In Aus einem Tagebuche werden drei alltägliche, geradezu unbedeutend erscheinende Erlebnisse geschildert, deren Gemeinsamkeit im Ausweisen der seelischen Bewirktheit des Willens liegt. Zuerst geschieht dies in Form der Schilderung der Unhintergehbarkeit eines»>Ohrwurms «:

»Ein unbedeutender, höchst uninteressanter Ausdruck aus einer Arie in einer Operette, den ich selbst nur vor ein paar Tagen von einem guten Freunde hörte, welcher ihn sich zu wiederhohltenmalen, aus Langerweile vorsang, kam mir heute Nachmittag, während dem ernsthaftesten Nachdenken, alle Augenblick, wider meinen Willen, in den Sinn, und ich konnte mich nicht enthalten, ihn mir ebenfalls zu wiederhohltenmalen vorzusingen, ohne den mindesten Gefallen daran zu finden « (Moritz, 1783, S. 36).

Ein jedem bekanntes Phänomen, das des »Ohrwurms «, soll beweisen, wie etwas Unbestimmtes, vom bewussten Denken und Wollen Getrenntes, den Willen als Voraussetzung für das Handeln des Autors bestimmt, insofern er sich nicht dagegen zur Wehr setzen kann. Dem ist entgegenzuhalten, dass, wenn sich ein »Ohrwurm « gegen den Willen geltend macht, die ihm dabei zugeschriebene Relevanz im Gegenteil geradezu seine Nichtigkeit im Hinblick auf die Bestimmung des Willens, genauer der Willensinhalte des betreffenden Subjekts und der aus ihnen resultierenden Handlungen, aufzeigt. Denn es ist äußerst fraglich, ob ein aufdringlicher »Ohrwurm « tatsächlich den Inhalt subjektiven Wollens lenken kann beziehungsweise das Handeln bestimmt oder nicht gerade nur dann wirksam ist, wenn es nicht um die Durchsetzung eines anderen Willensinhalts geht, sodass derselbe das Handeln gerade nicht stört. Es ist somit auch schwer zu glauben, dass die Melodie, die da in Moritz' Bewusstsein erschien, ohne dass er sich erklären kann, warum, ihn tatsächlich veranlasst haben sollte, sie gegen seinen Willen wirklich zu »singen « (sie also laut zu artikulieren). Denn das hieße, dass ein unwillkürlicher beziehungsweise unerwünschter Bewusstseinsinhalt dazu führte, am Willen vorbei und gegen ihn ein Handeln irgendwie zu erzeugen, dem man dann nicht mehr auskommt. Dies aber wäre etwa von der gleichen Absurdität, als hätte Moritz behauptet, die Melodie hätte ihn gegen seinen Willen und »ohne den mindesten Gefallen daran zu finden « dazu gezwungen, sein Haus zu renovieren. Und das liegt daran, dass ein Handeln ohne Willen oder gegen den Willen nicht nur theoretisch ein Widersinn ist. Mit dem Ziel, eine hinter dem individuellen Willen liegende psychische Determinante anhand der Unabweisbarkeit eines »Ohrwurms « zu beweisen, ist von einem konkreten Willensinhalt - nicht über die Arie nachzudenken - ein allgemeines Verhältnis des Subjekts zu sich selbst beziehungsweise zum Willen an sich und seinen Determinanten gestiftet. Dieses Muster zeigt sich in vergleichbarer Weise im nächsten Auszug aus demselben Tagebuchbericht: 
»Während dem Gehen gelang es mir, die Gedanken, die mich traurig machten, nach und nach zu unterdrücken. Es traten andre an ihre Stelle, welche sie verdrängten. Ich fand, wie klein und unbedeutend mein gegenwärtiger Verdruß im Verhältniß gegen meine Entwürfe sey. Diese Entwürfe rollten sich alle in meiner Seele auseinander, und der Gedanke an ihre wahrscheinliche Ausführung gewährte mir eine süße Täuschung. Dieß alles aber ereignete sich erst bei mir, nachdem ich eine Weile schnell gegangen war« (ebd., S. 37).

War es zuvor ein nicht willentlicher Bewusstseinsinhalt (der »Ohrwurm «), der vermeintlich das Wollen und Handeln bestimmte, so kommt in diesem Beispiel das Denken und Urteilen in seinem Zusammenhang mit dem Gefühl in den Blick. Und auch hier will Moritz zeigen, wie sein Urteil über eine Sache und das daraus resultierende Gefühl (Traurigkeit/Verdruss) nicht Ergebnis des bewussten Denkens, sondern eines (ihm) ganz fremden, von ihm getrennten Vorgangs ist (dem, des Gehens): Es kommt zum Ausdruck, dass - in Absehung des Beschlusses zum schnelleren Gehen das Gehen selbst ein Zustandsurteil hervorbringt. Nicht das gedankliche Urteil über ein Ereignis, nämlich dass es weniger traurig ist als zuvor gedacht, führt hier zur Relativierung des Verdrusses, sondern das Gehen bewirkt das Urteil. Betrachtet man näher, wie Moritz seinen Sinneswandel schildert, ergibt sich jedoch Folgendes: Der Autor denkt über einen Gegenstand nach und kommt zu einem Urteil, welches ihm das schlechte Gefühl des Verdrusses bereitet. Er fasst den Entschluss, dass er die schlechten Gefühle abwenden möchte und versucht deshalb, die Gedanken, die diese auslösen, zu unterdrücken. Während des längeren Gehens kommen ihm andere Gedanken zu einem ganz anderen Gegenstand (seine »Entwürfe «). Das Urteil über diesen Gegenstand (dass er sein Vorhaben in der Zukunft erfolgreich bewältigen werde) löst in ihm ein gutes Gefühl aus. Hernach setzt er beide Gegenstände ins Verhältnis zueinander und befindet jenen, der ihm schlechte Gefühle verursachte (ein weiteres Urteil) für weniger wichtig als den anderen. Diese schlichte Gedankenoperation, der Vergleich, ist ihm während des Gehens eingefallen, also, so Moritz' Schluss, muss sie durch das Gehen zustande gekommen sein. Dass ein körperlicher Vorgang Einfluss auf die Stimmung oder das Gefühlsleben haben kann, nimmt Moritz so fälschlicherweise als Beweis dafür, dass dieser auch das Denken seinem Inhalt nach bestimmte. Es zeigt sich jedoch, dass erstens die unterschiedlichen Gefühle, die Moritz jeweils schildert, seiner eigenen Schilderung zufolge je Ergebnis der Gedanken und Gedankenoperationen waren, die er vollzog, und dass zweitens auch die Inhalte der Gedanken, das heißt sowohl der Gegenstand und die Gedanken, die Moritz dazu hatte, als auch seine Entwürfe und das Urteil, das er über sie fällte, schon ganz unabhängig von seinem Spaziergang existierten. Und auch der Vergleich zwischen beiden Bezugspunkten, an dem Moritz schließlich ersteren relativiert und damit sein schlechtes Gefühl bekämpft, ist eine Leistung des Denkens und es ist überhaupt nicht einzusehen, wie das Gehen diese Leistung voll- 
bracht haben sollte. So beweist dieses Beispiel zwar nicht das, was Moritz beweisen wollte, nämlich dass das Denken und Urteilen durch ihm ganz fremde Vorgänge bestimmt sei, dafür zeigt es aber eindrücklich erstens, wie kompensatorisches Denken funktioniert, und zweitens, wie auch falsches Urteilen immer eine Sache des Denkens und seiner Fehler ist.

Im anschließenden Tagebuchausschnitt stellt der Verfasser nach einem Spaziergang in der Abendröte fest:

»Wie sehr hängt oft die ganze Richtung unsrer Gedanken von den äußern Gegenständen ab! Vielleicht ist es in dem Augenblicke, wo wir eine große Entschließung fassen sollen, kein unwichtiger Umstand, ob die Gegenstände, welche wir um uns her erblicken, roth oder grün sind « (ebd., S. 38).

Nach Moritz bestimmen somit nicht nur physische Vorgänge das Denken und Urteilen, sondern auch die äußeren Umstände, in denen es stattfindet. Damit ist gesagt, dass nicht das Bewusstsein beziehungsweise der Wille Gedanken entwickelt, sondern dass sie bedingt sind durch äußere Gegenstände, die auf innere Strebungen wirken. So sind weder die inneren noch die äußeren Determinanten der Gedanken konkret. Während die Wirkung innerer Determinanten nur in der behaupteten Abstraktion ihres Wirkens begriffen ist, sind die äußeren Determinanten ebenso abstrakt rot oder grün. Der Autor bebildert seine Idee an einer Entscheidungssituation, einer »große[n] Entschließung «, bei der er offenbar zwischen Alternativen zu wählen hat, von denen keine einen klaren Vorzug zu erkennen gibt beziehungsweise deren Folgen er jeweils zunächst nicht überblickt (anderenfalls wäre es keine Entscheidungssituation, sondern die Entscheidung wäre schon gefallen). Wie die Entscheidung schließlich ausfällt, betrachtet Moritz hier nicht als bewussten willentlichen Akt, der sich Gründen für oder gegen eine Seite verdankt, sondern er will zeigen, dass die Entscheidung von Umständen abhängt, die mit der Sache, auf die sie sich bezieht, überhaupt nichts zu tun haben, in diesem Fall der Sonnenuntergang. Dieser habe ihn nämlich veranlasst über die fragliche Sache anders zu denken. Eine gewisse Plausibilität erhält Moritz' Überlegung dadurch, dass sie schon in ihrer Anlage von einer Situation ausgeht, in der sich zwei gleichgewichtige, sich jedoch widersprechende Wünsche gegenüberstehen, sodass das Für und Wider beider Seiten sich wechselseitig aufhebt, durch ein Abwägen der Gründe also eine Entscheidung nicht ohne Weiteres herbeizuführen ist. Der Schluss, den Moritz dann zieht, zeigt jedoch weniger, dass Gedanken und Entschlüsse von der Farbe der Gegenstände respektive den äußeren Umständen abhängig sind, sondern offenbart vielmehr etwas darüber, wie der Autor mit der entsprechenden Situation umgehen will. Da er eine Entscheidung anhand von Gründen nicht treffen kann oder will, nimmt er einen weiteren Parameter zur Hilfe, nämlich die Stimmung, in der er sich gerade be- 
findet, und räumt ihr einen Einfluss auf die Alternativen ein. Diese fällt dann je nach Tageszeit oder Umgebung (Sonnenuntergang) unterschiedlich aus und soll dann mal für die eine, mal für die andere Seite sprechen. Dass die innere Stimmungslage jedoch genauso wenig eine Willensdeterminante ist, wie die äußerlich auf sie wirkenden Farben, erhellt schon daraus, dass Farben und Stimmungen in so einer Situation nie selbst eine Entscheidungsalternative entlang eines Inhalts erzeugen könnten, der nicht schon von vornherein zur Debatte stünde. Eine Alternative ist eben nur deshalb eine solche, weil man Gründe für sie geltend machen kann, und sie ist dementsprechend auch nur so gut (oder schlecht), wie die Gründe, die für (oder gegen) sie sprechen. Letztere werden jedoch nicht dadurch besser (oder schlechter), dass man in guter oder schlechter Stimmung ist, geschweige denn durch die Farben, mit denen man sich umgibt. Verlässt man Moritz' Ausgangspunkt der gleichwertigen Alternativen, so wird das besonders deutlich: Wenn jemand bei schönem Wetter lieber ins Schwimmbad geht als zu Hause abzuwaschen, so wäre es völlig absurd anzunehmen, die Farbe seiner Möbel oder der Häuser auf der Straße könnten oder würden seinen Willen zur anderen Alternative hin determinieren oder gar eine Dritte erzeugen, die es vorher noch nicht gab, wie etwa Stepptanz zu lernen. Die bezüglich Moritz' Zitat schwächere Lesart hinsichtlich der Willensdetermination wäre, sie im Sinne einer Empfehlung zu begreifen: nämlich dergestalt, dass man bei Entscheidungen auf die Farben der Umgebung achten sollte. Dies ließe dem Entscheidenden einen Handlungsspielraum, eine Einflusssphäre hinsichtlich des Ausgangs der Entscheidung. Diese Lesart setzt allerdings voraus, dass man erstens weiß, welche Farbe respektive welcher Umgebungseinfluss die Gedanken nach welcher Seite hin lenkt, und das unterstellt zweitens, dass man bereits eine Entscheidung für eine Seite getroffen hat und nun will, dass auch die Farben für diese Seite sprechen. Der geschilderte Fall zeigt also in beiden Varianten nicht, wie der Wille und das Denken des Menschen funktionieren, sondern gibt lediglich etwas Preis über den Willen des Autors, der sich dazu entschlossen hat, seine Entscheidung nicht von Gründen abhängig zu machen, sondern von seiner Stimmung, auf die die Umgebung dann einen Einfluss hat.

Die drei Beispiele zeigen somit, wie Moritz sich daran macht, die Seele zu erforschen. Im Duktus der unvoreingenommenen Empirie greift er zunächst alltägliche Situationen seines eigenen Lebens und Erlebens auf. Dann jedoch widmet er sich mitnichten der Analyse der von ihm gewählten Fälle, fragt gar nicht, wie sie zu erklären und welcher Schluss aus ihnen zu ziehen wäre, sondern legt vielmehr nahe, dass die Empirie schon für sich selbst spreche, also die Erklärung der Phänomene, die sie zeigt, bereits enthalte. Im Vorgeben, Erfahrungen schlichtweg zu schildern, liest Moritz so die für ihn daraus folgenden Schlüsse bereits in den Ausgangspunkt seiner Untersuchung hinein und unterstellt damit, dass seine Schlüsse inhaltlicher Bestandteil des durch ihn untersuchten Erfahrungsgegenstands sind. Alle drei Beispiele 
sollen zeigen, wie das Denken und Urteilen, das Wollen und Handeln ihrem Inhalt nach durch ihnen fremde, ganz äußerliche Momente bestimmt werden. Dass dieser Schluss aus den Beispielen selbst gar nicht folgt, vielmehr lauter Widersprüche erzeugt, hat die Analyse gezeigt. Für Moritz ergibt sich damit jedoch das eigentliche Rätsel: Er kennt die beiden Variablen, das bewusste Denken und Wollen und die sie scheinbar bestimmenden äußeren Faktoren, nimmt eine unbekannte Größe zwischen ihnen an, die Seele, und konstruiert dann einen Kausalzusammenhang zwischen den drei Bezugspunkten seiner Untersuchung. Nicht das bewusste Denken führt so zu einem Urteil und bestimmt danach den Willen, sondern die durch äußere Reize aktivierten unbestimmten inneren Strebungen bedingen das je bestimmte Urteil. Dieser Widerspruch lässt sich nur in der Abstraktion vom konkreten Urteil versöhnen, indem erstens von einem konkreten Urteil abgesehen und zweitens auf das Urteil als bedingtes Vermögen im Allgemeinen geschlossen wird. Mithin ist das Subjekt über (s)eine Negativbehauptung definiert: ihm widerfahren Dinge, das heißt sie finden ohne seine Absicht statt; aber gerade die Abwesenheit des Willens wird bei Moritz positiv aufgeladen, indem sie zu etwas anderem wird, das nicht nur existiert, sondern wirkt. Es zeigt sich auch, dass Moritz’ Beispiele nicht einfach zufällig gewählt sind, sondern vielmehr seine eingangs geschilderte Behauptung belegen sollen: Es gibt eine Seele, die sich der bewussten Kenntnis zunächst entzieht, aber das Bewusstsein und Handeln des Menschen maßgeblich bestimmt. Die Analyse hat jedoch gezeigt, dass Moritz seine These nicht durch entsprechende Beispiele belegt, sondern dieselbe vielmehr interessiert in sie hineinliest, indem er die Beispiele so auswählt und konstruiert, dass nichts anderes mehr aus ihnen zu folgen scheint als gerade seine ursprüngliche Behauptung.

\section{Das Resultat einer intakten Seelentätigkeit: Der pflichtbewusste Weltbürger}

In »Ueber den Zweck der Thränen « sucht Gruner (1791), Autor des Magazinbeitrags, die Vernunft der Natur nachzuweisen:

»Wenn aber der Traurige weint, so wähnt er so oft, dadurch den verlornen Gegenstand noch zu ehren; und wenn er zu weinen aufgehört hat, so fühlt er sich leichter; indem also der Leidende in der Meinung steht, er leiste dem geliebten Gegenstande noch die letzte Pflicht, so weiß er nicht, daß die Natur ihn dazu zwingt, daß durch seine Thränen, die Vorstellung an den vermißten Gegenstand in ihm nach und nach verloren geht, und daß er selbst wieder in den Gemüthszustand gesetzet wird, welcher zur Erfüllung seiner Pflichten als Mensch und Weltbürger am zuträglichsten ist « (S. 22). 
Übereinstimmend mit Moritz' Ausgangsthese, dass das Dunkel über den Zusammenhang zwischen körperlicher Daseinsform und Seelenleben des Menschen und dessen Stellung in der Welt im Dienste der Wahrheitsfindung über den Menschen wissenschaftlich erhellt werden müsse, identifiziert auch Gruner die gesunde Seele mit der Funktionalität des Subjekts in seinem gesellschaftlichen Zusammenhang (»Erfüllung seiner Pflichten «). Er misst also umgekehrt seelische Störungen und Krankheit an subjektiver Dysfunktionalität. Und wieder: Wenn die innere Natur der äußeren Bürgerpflicht folgt, ist äußeren Anforderungen eine Unhintergehbarkeit attestiert, die ob ihrer Entsprechung zur Natur legitim ist. Somit ist erneut der Gedanke eingeholt, das Wesen der Seele entspreche im Prinzip beziehungsweise qua Natur dem der Gesellschaft. Diese wiederum ist gefasst nicht als eine Ansammlung normativer Forderungen und Erwartungen an das Individuum, an denen es sich abarbeitet oder scheitert, sondern als seine verobjektivierte und verdinglichte innere Natur. Am Beispiel des Weinens will der Autor dies zeigen, und zwar, indem er das zu Beweisende im Beweis bereits unterstellt. Gruner fasst die Traurigkeit zielstrebig nicht als das auf, was sie ist, sondern identifiziert diesen »Gemüthszustand « gleich ganz mit dem, was ihn daran interessiert, nämlich mit seinem Effekt auf das Individuum. Das Weinen wiederum verschafft dem Traurigen Erleichterung, er wird wieder handlungsfähig, kann also seine Pflichten erfüllen. Und das begrüßt Gruner so sehr, dass er die Funktion unmittelbar für die Sache selbst hält. Also, folgert er, muss das Weinen eine Tätigkeit der Natur sein, die den Menschen wieder seine Pflichten erfüllen, also zu sich selbst kommen lässt. Davon weiß das Subjekt Gruners Implikation folgend jedoch nichts, denn neben dieser potenziellen Identität zwischen innen und außen wirken Kräfte, die dem Subjekt Handlungsfähigkeit vorspiegeln. Diese Vorspiegelung ist jedoch nur Produkt des Zusammenspiels äußerer Reize, die auf innere Kräfte wirken, welche ihrerseits, weil sie im Subjekt stattfinden, als Ausdruck seiner bewussten Bezugnahme auf die äußeren Reize erscheinen. Tatsächlich sind sie jedoch Ausdruck irrationaler Kräfte.

»Uebrigens untersuchten wir hier auch nur, was die Natur durch die Thränen bei dem Traurigen bewirken läßt; und in dieser Rücksicht verdient noch die allgemeine Erfahrung bemerkt zu werden; daß der Mensch vielmehr leidet, dessen Körperbau die leichtere Ergießung der Thränen verhindert. Es ist Trieb der Natur, des Schmerzes loß zu werden, und hier wird die Natur daran gehindert. Die Gehirnfibern, die die unangenehmen Vorstellungen erwecken, sind reger wie jede andere, und maßen sich Alleinherrschaft an « (ebd., S. 23).

In dieser Vorstellung ist der Mensch dreifach bewirkt, erstens durch irrationale »Gehirnfiber «, zweitens durch eine vernünftige Natur und drittens durch die der Natur potenziell entsprechende Außenwelt. 


\section{Ein Fazit}

»Erkenne dich selbst! « Unter diesem Motto machte sich Karl Philipp Moritz gemeinsam mit anderen Autoren daran, systematisch und vorurteilslos die Seele zu erforschen, und zwar durchaus im Geiste der Zeit. ${ }^{4}$ Doch wie erkennt und was erfährt er eigentlich, »der Mensch «, wenn er sich selbst betrachtet? Oder anders gefragt: Wie kommt man von der empirischen Beobachtung mit dem Ziel, die Psyche ihrem Wesen nach zu erkennen, zu einer Verhältnisbestimmung, in der diese sich gleich zweimal äußert: einmal als bisherige Verhinderung und einmal als potenzielle Ermöglichung des Ideals einer moralisch verfassten Gemeinschaft? Ist damit ein blanker Erkenntnisbedarf formuliert oder bereits ein Vorurteil, das einen interessierten Blick nach sich zieht?

Die in den vorangegangenen Punkten entwickelte Analyse hat zeigen wollen, dass das im Magazin zur Erfahrungsseelenkunde angekündigte Forschungsvorhaben, die hinter dem bewussten Denken und Handeln des Individuums liegenden, es jedoch konstituierenden Seelenprozesse aufzufinden, schon im Ausgangspunkt eine Unterstellung enthält - nämlich die, dass es solche Seelenprozesse und damit den ganzen in Rede stehenden Gegenstand überhaupt gibt. Es hat sich gezeigt, wie dieser nicht erst bewiesen, sondern über die rhetorische Figur des Vergleichs gestiftet wurde, der dann auch für den Inhalt der Seelentätigkeit eine enorme Produktivkraft bewies. Über die darin behauptete Gesundheit beziehungsweise Krankheit der Seele und ihre Identifikation mit den gesellschaftlichen Phänomenen des Rechts erhält die Seele ein Sollen, einen moralischen Imperativ und in ihrer moralischen Natur ein Entsprechungsverhältnis zu einer gedachten idealen Gesellschaft. In dem schlichten Dreischritt: gesunde Seele $=$ funktionales Subjekt $=$ Erfüllung der gesellschaftlichen Pflicht erklärt Moritz dann umgekehrt auch, und zwar unter Abstraktion von allen wirklichen gesellschaftlichen Charakteren der Individuen, eine Abweichung von den rechtlichen Normen als schadhaften und zu heilenden Abweg von der eigenen Natur - und immunisiert so die gesellschaftlichen Vorgaben gegen Kritik, die die gesellschaftlichen Institutionen selbst infrage stellt. Zugleich wird damit klar, was die Seele höchst selbst zu ihrer Heilung gebietet: jene besteht dementsprechend darin, das Individuum wieder zu seiner gesellschaftlichen Funktion zu befähigen beziehungsweise es mit dieser zu versöhnen. Den dann folgenden Erfahrungsberichten wird folglich nicht etwa der Inhalt der Seelentätigkeit entnommen, sondern sie geraten vielmehr zu einem Beweisverfahren der Existenz der Seele überhaupt beziehungsweise zur Konstruktion von Determinanten des bewussten Denkens und Handelns, aus denen dann auf die Seele als unbekanntes inneres X geschlossen wird. Die darin eingeschlossene Relativierung des freien Willens macht so Platz für seinen besagten moralischen Inhalt.

Von diesem Resultat her ließe sich folgern, die Autoren des Magazins müssten in ihrem Ausgangspunkt gerade auf einen Gegensatz zwischen Individuum und Gesellschaft 
gestoßen sein: So fassen auch Jaeger und Staeuble als Paradigma des Magazins »die Verständigung von Autoren und Lesern über Erfahrungsformen des Leidens an der Gesellschaft « (Staeuble, 1985, S. 26) und als »Schwierigkeiten [...] einer Situation der Auflösung der festgefügten ständischen Ordnung und des Übergangs zu einer neuen Ordnung « (Jaeger \& Staeuble, 1978, S. 76). Hinzuzufügen ist dieser Feststellung jedoch, wie gezeigt, dass dieser Gegensatz bei Moritz über die in Aussicht gestellte potenzielle Identität zwischen gesunder Seele und moralischer Gesellschaft ideell eine Auflösung findet. Und zwar von den Schwierigkeiten des Individuums weg und zu einer Idealisierung gesellschaftlicher Normen beziehungsweise einem Ideal der Entsprechung zwischen Individuum und Gesellschaft hin - mit ihrem Grund in der menschlichen »Natur «.

Das Magazin zur Erfahrungsseelenkunde wurde von seinem Hauptherausgeber Moritz in der Zeitschrift Deutsches Museum als ein Vorschlag vorstellig gemacht; von einem sicheren Verständnis der menschlichen Psyche wurde entsprechend noch keineswegs ausgegangen. Es galt, wie die Analyse gezeigt hat, zunächst ein Forschungsvorhaben zu begründen, und wenngleich hinter die von vornherein feststehende Behauptung jener Eigengesetzlichkeit namens Psyche trotz aller Begründungswidersprüche nicht zurückgetreten wurde, so lassen sich die Beiträge unter den Zweck einer offenbar noch für nötig befundenen Beweisführung fassen. Inwiefern das hier rekonstruierte psychologische Denkmuster, auf dessen Geläufigkeit einleitend Bezug genommen wurde, zum geteilten Wissen der Gegenwartspsychologie(n) gehört, wäre eine naheliegende Forschungsfrage. Denn inwieweit und mit welcher inneren Logik der menschliche Wille durch Triebe, Kräfte, kognitive wie neuronale Prozesse, die Umwelt oder multikausal zum nur noch relativen Grund der Erklärung menschlichen Handelns hergenommen wird, lohnte sich allemal zu prüfen. ${ }^{5}$

\section{Anmerkungen}

1 Um zwei möglichen Missverständnissen vorzubeugen: Wenn hier, erstens, von »Psyche« oder »der Psyche« die Rede ist, dann wird dieses Wort nicht zur Bezeichnung einer irgendwie in der Welt existenten Sache verwendet; dementsprechend will der Text nicht den Psyche-Begriff, seine Varianten, Entwicklungen oder sein Verschwinden etc. diskutieren, sondern auf eine in der im weiten Verständnis des Wortes Psyche enthaltenen Frage nach dem Warum menschlichen Treibens aufmerksam machen. Der Text nimmt sich vor, an Moritz' Erfahrungsseelenkunde eine Begründungslogik beziehungsweise ein Denkmuster aufzuzeigen, das in verschiedensten, auch ganz modernen Deutungen menschlichen Handelns aufscheint und ihnen zugrunde liegt. Diese Logik enthält die Annahme von etwas Allgemeinem am Menschen, das ihn neben seinen biologischen Voraussetzungen und hinter seinen je konkreten Zwecken und Gründen auch noch ausmacht; sie sucht also eine Rationalität, die in systematischen Antworten auf die Frage besteht, warum Menschen Denken und Handeln wie sie Denken und Handeln. Sooft das Wort Psyche oder Seele in der hier geschilderten Weise verwendet wird, handelt es sich also um eine Negativbestimmung; es ist von keiner Sache die Rede, sondern von einer zu findenden Sache, einer 
zu füllenden Leerstelle, die dem benannten Denkmuster inhärent ist. Wenn im Text, zweitens, behauptet wird, die Existenz der Psyche würde selten bezweifelt, dann soll damit nicht gesagt sein, dass es niemanden gäbe, der sie bezweifelt, sondern es soll darauf hingewiesen werden, dass das zu zeigende Begründungsmuster in zahlreichen Variationen sehr weit verbreitet ist.

2 Üblicherweise wird der Beginn der Psychologie als eigenständige Wissenschaft auf die Eröffnung des Wundt'schen Labors 1879 in Leipzig datiert (Schwarz \& Pfister, 2016). Geht man allerdings von der Anzahl psychologischer Vorlesungen, Lehrbuchveröffentlichungen und Zeitschriftengründungen Ende des 18. Jahrhunderts aus, »dann könnte die Psychologie bereits [ca. 100 Jahre früher] für ein selbständiges Fach, zumindest aber für eine starke Disziplin innerhalb der philosophischen Fächer gehalten werden« (John, 2002, S. 167). Sie entwickelte sich unter dem Dach der philosophischen Fakultät um 1800 als empirische Erfahrungswissenschaft schrittweise zu einer eigenständigen Disziplin (Eckardt et al., 2001). Diese Feststellung soll den hier nicht weiter zu verfolgenden Hinweis geben, dass der historische Kontext mit der Formulierung des Gegenstandes der Psychologie auf unterschiedlichen Ebenen zusammenhängt. So hätten die politökonomischen Veränderungen im Übergang zur Moderne systematische Problemlösungsnotwendigkeiten empirischer Subjektivität hervorgebracht (Jaeger \& Staeuble, 1978) und die sich seinerzeit funktional herausbildenden Disziplinen durch ihre systematisch veränderten Weisen der Wissensproduktion selbst Veränderungen am Wissen erzeugt (Stichweh, 1984). Nicht zuletzt habe sich an Moritz' Erfahrungsseelenkunde das »Interesse an einer neuen Psychologie aus[gedrückt« (Spies, 1992, S. 122), und zwar um »die Anstrengung der Aufklärung auf das aufzuklärende Subjekt selbst auszuweiten, als Forschungsinteresse, das endlich ernst zu nehmen sei« (ebd.). In diesem Zeitraum ändert sich also die Tradition der Seelenforschung grundlegend.

3 Diese potenzielle Entsprechung zwischen Individualpsyche und gesellschaftlichen Verhältnissen (deren Logik auch schon im vorangegangenen Kapitel anhand des Verbrechers, auf dessen psychische Abweichung von der rechtlichen Verfehlung geschlossen ist und mithin umgekehrt, indem aus der kritischen Feststellung seiner Existenz als Schaden aller auf die Notwendigkeit einer Wissenschaft der Individualseele gefolgert wird) steht nicht im Widerspruch zu aufklärerischer Gesellschaftskritik. Denn individuelle Verfehlungen, die zum Beweis gesellschaftlicher Missstände (vgl. Moritz' psychologische Forschungsrelevanz) herangezogen werden, stellen die Möglichkeit eines Gelingens gesellschaftlichen Miteinanders nicht prinzipiell infrage, im Gegenteil: Die Potenz eines harmonischen Miteinanders bleibt durch den Rekurs auf die individuelle Abweichung davon gerade erhalten. So kritisiert Moritz die Missstände im Lichte einer geschädigten/potenziell gesunden Psyche, weshalb er auch als Gegenmittel die Erforschung des Individuums identifiziert. In dem Sinn ist die »Erfahrung des Leidens an der Gesellschaft« (Jaeger \& Staeuble, 1978, S. 72) zentrales Thema der Erfahrungsseelenkunde. Darin sind die Individuen auch keineswegs passiv, sondern kommen in ihrer psychischen »Selbsttätigkeit« (ebd., S. 79), in ihrem geistigen Umgang mit den widersprüchlichen Verhältnissen des Übergangs von der Feudalgesellschaft zur bürgerlichen Gesellschaft vor. Den Anspruch auf Anerkennung, den das Individuum von dort aus für seine Individualität erhebt und von dem ausgehend es die gesellschaftlichen Strukturen als dieser entsprechend billigt oder als von ihr abweichend kritisiert, beschreibt Spies (1992) folgerichtig an Moritz' Roman Anton Reiser und kommt zu dem Schluss, dass »die Anklage des Elends der psychologischen Perspektive untergeordnet [bleibt]《 (S. 156).

4 »Der Schriftsteller und Pädagoge Moritz nahm mit diesem Problemhorizont keineswegs eine Sonderstellung in der Aufklärungsbewegung ein. Im letzten Drittel des 18. Jahrhunderts war als Bewußtseinslage präsent und wurde als Thema und Aufgabe u. a. von philosophischer, anthropologischer und literarischer Seite formuliert, daß Aufklärung die Aufklärung des Menschen über sich selbst einzubeziehen hatte (Kaufmann, 1995, S. 48). 
$5 \quad$ Vor dem Hintergrund eines emanzipatorischen Subjektverständnisses befasst sich damit die Kritische Psychologie: »Klagen über die Lebensferne, Sinnfremdheit und Subjektverkennung der Psychologie durchziehen die Geschichte unserer Disziplin. Schon um die Jahrhundertwende bilanzierte William Stern (1900), daß die psychologische Arbeit des 19. Jahrhunderts wesentlich eine ssubjektlose Psychologier hinterlassen habe. Dieser Kritiktopos hat seither, mit dem Siegeszug des Funktionalismus/Behaviorismus, aber nicht minder nach der erklärten ’kognitiven Wendes, mit der Konjunktur der Computermetapher, dem Vormarsch der Neuropsychologie etc. Bestand. Die etablierte Psychologie, heißt es ein um das andere Mal, betreibe begrifflich und methodisch Subjektverleugnung und bewege sich damit andauernd im Selbstwiderspruch, da sie Theorien konstruiere, in denen der Mensch als durch die Gegebenheiten seiner Umwelt determiniert angesehen werde, während sich die Theoriekonstrukteure selbst als autonom und eigenaktiv erführen« (Maiers, 1996, S. 167).

\section{Literatur}

Eckardt, G., John, M., van Zantwijk, T. \& Ziche, P. (2001). Einleitung: Ansätze einer Entwicklung der Psychologie zur empirischen Wissenschaft um 1800. In G. Eckardt, M. John, T. van Zantwijk \& P. Ziche (Hrsg.), Anthropologie und empirische Psychologie um 1800. Ansätze einer Entwicklung zur Wissenschaft (S. 1-20). Köln et al.: Böhlau.

Gruner, J.E. (1791). Über den Zweck der Thränen. Aus einer ungedruckten Schrift über den Trost. In K.P. Moritz (Hrsg.), Gnothi sauton oder Magazin zur Erfahrungsseelenkunde als ein Lesebuch für Gelehrte und Ungelehrte. In P. Nettelbeck \& U. Nettelbeck (Hrsg.) (1986), Karl Philipp Moritz. Die Schriften in dreißig Bänden (Bd. VIII, St. 1) (S. 20-23). Nördlingen: Franz Greno.

Jaeger, S. \& Staeuble, I. (1978). Die Gesellschaftliche Genese der Psychologie. Frankfurt/M.: Campus.

John, M. (2002). »Empirische Psychologie« im System der Wissenschaften um 1800. Psychologie und Geschichte, 10(3), 166-177.

Kaufmann, D. (1995). Aufklärung, bürgerliche Selbsterfahrung und die»Erfindung «der Psychiatrie in Deutschland, 1770-1850. Göttingen: Vandenhoeck \& Ruprecht.

Maiers, W. (1996). Der Subjektbegriff der Kritischen Psychologie. In M. Heinze \& S. Priebe (Hrsg.), Störenfried »Subjektivität». Subjektivität und Objektivität als Begriffe psychiatrischen Denkens (S. 167-221). Würzburg: Königshausen \& Neumann.

Moritz, Karl Philipp (1782). Vorschlag zu einem Magazin einer Erfahrungsseelenkunde. Deutsches Museum, 1, 485-503.

Moritz, K.P. (1783). Gnothi sauton oder Magazin zur Erfahrungsseelenkunde als ein Lesebuch für Gelehrte und Ungelehrte. In P. Nettelbeck \& U. Nettelbeck (Hrsg.). (1986). Karl Philipp Moritz. Die Schriften in dreißig Bänden (Bd. 1, St. 1) (S. 7-8). Nördlingen: Franz Greno.

Schwarz, K.A. \& R. Pfister (2016). Scientific Psychology in the 18 $18^{\text {th }}$ Century: A Historical Rediscovery. Perspectives on Psychological Science, 11, 399-407.

Spies, B. (1992). Politische Kritik, Psychologische Hermeneutik, Ästhetischer Blick. Die Entwicklung bürgerlicher Subjektivität im Roman des 18. Jahrhunderts. Stuttgart: J. B. Metzler.

Staeuble, I. (1985). sSubjektpsychologier oder ssubjektlose Psychologie - Gesellschaftliche und institutionelle Bedingungen der Herausbildung der modernen Psychologie. In M. G. Ash \& U. Geuter (Hrsg.), Geschichte der deutschen Psychologie im 20. Jahrhundert. Ein Überblick (S. 19-44). Opladen: Westdeutscher Verlag.

Stichweh, R. (1984). ZurEntstehung des modernen Systems wissenschaftlicher Disziplinen: Physikin Deutschland 1740-1890. Frankfurt/M.: Suhrkamp. 


\section{Die Autorin}

Monique Lathan, M.Sc., Institut für Soziologie der Martin-Luther-Universität HalleWittenberg (MLU). Arbeitsschwerpunkte: psychologische Theorieentwicklung/psychologische Subjektdeutungen.

Kontakt: monique.lathan@soziologie.uni-halle.de 